\title{
Sinergias entre instituciones socioeducativas como respuesta ante las necesidades sociales. Una aproximación a través del análisis de redes sociales
}

Recibido: 5 de febrero de 2019 / Revisado: 16 de febrero de 2019 Aceptado: 1 de abril de 2019 / Publicado: 22 de julio de 2019

IRENE MORENO-MEDINA Departamento de Didáctica y Teoría de la Educación, Universidad Autónoma de Madrid, España irene.morenom@uam.es

CARMEN MÁRQUEZ VÁZQUEZ Departamento de Trabajo Social y Servicios Sociales, Universidad Pablo de Olavide, España cmarvaz@upo.es

\section{RESUMEN}

Las crisis sociales y económicas que han vivido y se viven en el madrileño barrio El Pozo-Entrevías (en el distrito de Puente de Vallecas) ha supuesto una transformación en la forma de relacionarse por parte de las entidades socioeducativas que se encuentran en él. El objetivo de este estudio es conocer y analizar cómo son las relaciones que existen entre las entidades seleccionadas como representativas de instituciones socioeducativas: un centro de educación infantil y primaria, un centro de educación secundaria y una ONG.

Para describir y analizar dichas relaciones, se ha utilizado una metodología mixta apoyada en entrevistas semiestructuradas y hemos utilizado como metodología innovadora en educación, el Análisis de Redes Sociales (ARS), el cual nos permite analizar y comprender los vínculos de apoyo interinstitucionales. El análisis se realizó en torno a cuatro categorías: tipo de relación

\section{ABSTRACT}

Synergies between socio-educational institutions as an answer to social needs. An approximation through social networking analysis.

The social and economic crises that have lived and are lived in El Pozo-Entrevías (in the district of Puente de Vallecas, Madrid) has meant a transformation in the way of relating by the socio-educational entities that are in it. The objective of this study is to know and analyse the relationships that exist between the entities selected as representative of socio-educational institutions: a centre for early childhood and primary education, a secondary education centre and an NGO.

To describe and analyse these relationships, a mixed methodology based on semi-structured interviews has been used and we have used the Social Network Analysis (SNA) as an innovative methodology in education, 
formal o informal, frecuencia de los intercambios, antigüedad y tipo de apoyo que intercambian. Las primeras aproximaciones de esta investigación piloto muestran que existen fuertes redes entre los centros educativos y las asociaciones como respuesta a las necesidades de un contexto caracterizado por enfrentarse a unas condiciones socioeconómicas desafiantes.

Palabras Clave: análisis de redes, escuela, escuela desfavorecida, cambio social, Organización no gubernamental which allows us to analyse and understand inter-institutional support links. The analysis was carried out around four categories: type of formal or informal relationship, frequency of exchanges, seniority and type of support that they exchange. The first approximations of this pilot research show that there are strong networks between schools and associations in response to the needs of a context characterized by challenging socioeconomic conditions.

Keywords: Network analysis, school, Disadvantaged schools, Social change, Nongovernmental organizations

\section{Introducción}

La crisis social, económica y humanitaria que acontece en nuestra sociedad está incrementado la brecha de la desigualdad social (Stiglitz, 2012). Por primera vez, existe un riesgo real de que la actual generación de jóvenes acabe teniendo peores condiciones de vida que sus padres y madres (Comisión Europea, 2017). Ante este panorama, la educación se posiciona como un instrumento definitivo en las políticas orientadas a la reducción de desigualdades sociales. La educación ofrece a todas las personas una oportunidad de mejora de las posibilidades de acceso al mercado laboral, a la cultura y a la participación social (Blanco, 2006). No obstante, no podemos obviar que el acceso a la educación no siempre está garantizado en igualdad de condiciones y que los estudiantes que provienen de contextos socioeconómicos desafiantes son más vulnerables al fracaso o al abandono escolar (López, D’Alessandre y Pla, 2006). Estos estudiantes enfrentan a un mayor número de barreras familiares y sociales que se traduce en un escaso apoyo para finalizar, con éxito, la escolarización obligatoria. Algunos de los centros educativos ubicados en barrios desfavorecidos han comenzado a desarrollar estrategias de coordinación y apoyo para optimizar los recursos que poseen y atender así las necesidades de un mayor número de estudiantes.

\subsection{Escuelas en contextos socioeconómicamente desafiantes}

Los contextos socioeconómicamente desafiantes, vulnerables o empobrecidos tienen características que los lleva a encontrarse en una situación de riesgo en la que ninguna de las medidas tomadas para mejorar sus condiciones ha resultado y han seguido en un estancamiento sociocultural y económico. Según Corraliza (2014) los barrios vulnerables son aquellos en los que hace falta, urgentemente, luchar contra la exclusión social y la degeneración urbana. Basándonos en términos sociológicos, Alguacil 
(2011) refiere que la exclusión social y la exclusión residencial son determinantes para que un contexto sea vulnerable. Esta vulnerabilidad supone, además, un aumento de las amenazas y riesgos que afectan a las personas, grupos de personas, etcétera, así como el debilitamiento de los mecanismos para hacer frente a dichas amenazas y riesgos (Alguacil, Camacho y Hernández, 2014).

Los barrios que se encuentran en contextos socioeconómicos más desfavorecidos tienen diferentes características (O’Connor et al., 1999), cuentan con menos ayudas públicas, la prensa local y nacional desprestigia el barrio, hay múltiple cambio del personal que trabaja en él, viven personas en extrema pobreza, etcétera.

Desde la comunidad científica también se ha tenido interés por investigar para conocer y describir las características de las escuelas ubicadas en contextos socioeconómicamente desafiantes (Harris, 2002; Harris y Chapman, 2004; Chapman y Harris, 2004; Thrupp y Lupton, 2006; Kauppinen, 2008; Thrupp, 2011) y para analizar las dinámicas que se generan en estos espacios y poder aportar respuestas, desde el ámbito educativo, ajustadas a sus necesidades (Beckett y Wrigley, 2014; Hayes, Jonhston y King, 2009; Horgan, 2009; Smith, 2014). Muchos de estos trabajos han asegurado que la mejora de las escuelas en contextos desafiantes requiere de un mayor apoyo que provenga de fuera de las mismas (Potter et al., 2002; Stoll y Myers, 1998).

En España, los centros educativos ubicados en lugares socioeconómicamente desafiantes se enfrentan, además de a las limitaciones propias, a dificultades derivadas de un sistema educativo que no afronta la segregación y exclusión socioeconómica (Murillo y Martínez-Garrido, 2018). Prueba de ello, es que el sistema educativo español ha pasado en pocos años de ser uno de los más equitativos del mundo a sufrir graves inequidades por razones socioeconómicas (PISA, 2014). También Gray (2000) reconoce que en las zonas más empobrecidas, la concentración de escuelas que acumulan desventajas es mayor, circunstancia que se relaciona directamente con el rendimiento educativo de sus estudiantes. Los docentes que se encuentran en estas escuelas admiten que no poseen la capacidad ni competencias suficientes para poder intervenir con el alumnado y sus familias (Corbett, Wilson y Williams, 2002; Dyson, 2004). Y las familias ven agravada su situación debido a otro elemento que fortalece el distanciamiento social, cultural y económico que viven y es la segregación que produce la educación privada (Murillo, Belavi y Pinilla, 2018).

La acumulación de todas estas situaciones favorece los procesos de selección que realizan algunas escuelas no públicas para controlar el perfil del alumnado que accede a ellas. Estos procesos mantienen al margen al alumnado de familias ubicadas en contextos desafiantes fomentando, más si cabe, la segregación que ya sufrían por estar alojadas en contextos más empobrecidos.

Muchas de las escuelas públicas ubicadas en entornos desafiantes plantean dificultades particulares relacionados con sus situaciones de pobreza (Fernández, Tuset, Pérez y García, 2013) que generan ne- 
cesidades no cubiertas por la administración educativa. Esto las obliga a permanecer en una constante búsqueda de recursos de apoyo externos para hacer frente a las necesidades de su alumnado. Muchos de estos recursos proceden directamente de la propia comunidad, del grupo de personas que próximas al centro, mantienen interacciones recíprocas y se identifican con un sistema de creencias similar (Nancy, 1991 y 2000). Otras veces, los recursos de apoyo proceden de programas y actuaciones puesto en marcha por organizaciones y movimientos sociales (Lomnitz,1977; Sipi, 2000).

Todos estos factores propician que, en algunos casos, las escuelas acudan a su propia red institucional o de relaciones para poder dar cobertura a sus necesidades. La relación entre estas instituciones socioeducativos nace de la falta de apoyo institucional y el liderazgo activo de equipos directivos que se ven forzados a establecer nuevos lazos para poder cubrir las necesidades detectadas. Estas relaciones entre instituciones se crean gracias al compromiso de las personas que las representan.

Cuando las relaciones que unen a los individuos, que conviven en un mismo contexto, tienen un mismo objetivo hablamos de redes locales o territoriales (Civís y Longás, 2015). En esta línea, el barrio puede ser descrito como una red de relaciones entre instituciones de diversa naturaleza en las que la escuela es un actor más de la estructura comunitaria (Pereda, 2003). En dicha estructura las redes sociales son fuente de recursos emocionales, informacionales y materiales que mejoran la calidad de vida de sus miembros (Paredes et al., 2015). Como se ha avanzado, anteriormente, en un barrio pueden simultanearse diferentes tipos de relaciones. En nuestro caso de estudio, son las relaciones socioeducativas locales las que centran nuestra atención. Estas relaciones han sido definidas como:

Un modelo de organización alternativo a los tradicionales organigramas jerárquicos, con capacidad para integrar instituciones y actores con responsabilidad educativa en un plano de corresponsabilidad, unidos por el interés común, primero para compartir el análisis de las necesidades y los proyectos de un territorio o comunidad, y segundo para coordinar la acción socioeducativa de un modo integral y coherente (Longás, Civís y Riera, 2008, p.306).

\subsection{Análisis de redes en contextos educativos}

El ARS es una herramienta teórico-metodológica que nos permite avanzar en la comprensión de los fenómenos sociales mediante la observación de las interacciones individuales e institucionales en determinados contextos y de las que emergen las estructuras sociales (Lugo-Marín, 2011). Su unidad básica de análisis es la red social, entendida como una estructura colectiva entre actores vinculados por interdependencias de amistad, parentesco, intercambio o intereses comunes (Matilla-García, 2017). Cada uno de estos actores es considerado un nodo central de la red (Sanz, 2003). 
El ARS nos permite analizar y describir las estructuras relaciones a varios niveles. Por un parte, permite describir las propiedades particulares de la red tales como las características estructurales y contextos en las que se producen y persisten (Luke, 2005). Por otro lado, facilita la comprensión de los efectos colectivos que se producen a partir de las características de la red, como difusión de la información o relaciones de poder (Requena, 1989).

Aunque es una metodologías más aplicada y desarrollada en otros campos de investigación alejados del ámbito educativo, tales como inmigración, sanidad o empresa (Gualda y Márquez, 2010; Zazo, Ardines y Castro, 2015) puede tener un gran impacto en el ámbito educativo por su carácter innovador. Hasta el momento, se destacan la contribución del ARS al análisis de la influencia de las relaciones que mantiene los estudiantes sobre el rendimiento académico (Gremmen et al., 2017; Shin y Ryan, 2014). También ha sido objeto de análisis por parte de los investigadores de redes, la diferencia en la cohesión estructural de sus redes (Santos, 2010). Por otra parte, los motivos por los que los estudiantes establecen relaciones de amistad o de discriminación social en las aulas, entendida como la preferencia relativa por las relaciones intraétnicas sobre las interétnicas de los estudiantes, también han sido analizadas en los contextos educación secundaria holandeses (Vermeij, van Duijnb y Baerveldt, 2009). Este último trabajo destaca que los estudiantes de la mayoría étnica no mostraban interés por relacionarse con los estudiantes de las minorías, aunque tampoco evidenciaban actitudes discriminatorias. En cambio, los propios estudiantes pertenecientes minorías étnicas establecían mayor número de relaciones con miembros de otras etnias, pero también mostraban actitudes más discriminatorias. Por último, el ARS ha constatado una significativa diferencia de género en los criterios educativos para establecer relaciones con los iguales.

\section{Metodología}

La descripción y análisis de las relaciones que mantienen las tres instituciones educativas, objetivo de nuestro trabajo, se ha realizado combinando una metodología mixta basada en el análisis de redes sociales y entrevistas semiestructuradas.

De manera presencial, se realizaron entrevistas a los representantes de las instituciones escolares del barrio Entrevías (Madrid). El barrio nació en 1950 con la llegada de trabajadores emigrantes que provenían de otras zonas rurales de España. Desde sus inicios ha presentado una importante delimitación tanto física como social. Urbanísticamente, el trazado del ferrocarril, los accesos a la ciudad de la M-30, el parque y una gran avenida demarcan su ubicación y separan a su población del resto de barrios vecinos. Tanto es así que, aunque administrativamente, el barrio Entrevías se reconoce como un único barrio, los y las vecinas declaran un manifiesto sentimiento de pertenencia que los vincula a Entrevías en un extremo y a El Pozo en el otro. 
Socioeconómicamente, ha presentado unas de las mayores tasas de desempleo de la ciudad y los niveles socioeconómicos y culturales más bajos, según datos ofrecidos por el Ayuntamiento de Madrid. Por el contrario, en el Pozo se percibe una gran tradición de colaboración vecinal como forma de lucha social y consecución de derechos básicos que ha sustentado la creación de un gran tejido asociativo. Ejemplo de ello, es la cooperativa eléctrica de El Pozo quienes llevaron por primera vez la luz al barrio (López de Lucio, 1988) y todavía hoy dan servicio a la comunidad. Toda esta casuística fue considerada para la elección del contexto de estudio y motivaron la decisión de centrar la investigación en las instituciones socioeducativas con sede en El Pozo.

Por su naturaleza, las entrevistas se realizaron al profesional responsable de la dirección del centro educativo de educación infantil y primaria, de la jefatura de estudios en los centros de educación secundaria o a la persona responsable de la coordinación en las asociaciones sin ánimo de lucro. Esto permitido ampliar la comprensión de las relaciones interinstitucionales que mantienen.

La muestra de entidades seleccionada pertenece al barrio de Entrevías-El Pozo (distrito Puente de Vallecas de Madrid, España). En este trabajo se presentan los primeros datos de una investigación que continua en curso y que se irá ampliando paulatinamente. A continuación, presentamos las tres instituciones socioeducativas elegidas para comenzar esta investigación y que, para proteger su identidad, han sido anonimizadas.

- CEIP: Centro de Educación Infantil y Primaria con una única línea. Cuenta con programas para el desfase curricular y compensación para los/as alumnos/as con necesidades específicas de apoyo educativo.

- IES: Instituto de Educación Secundaria Obligatoria, Bachillerato y Educación de Adultos. Tiene tres líneas de ESO y las modalidades de bachillerato con las que cuenta son Ciencias y Humanidades y Ciencias Sociales.

- ONG: organización no gubernamental independiente y laica que cuyo trabajo se realiza a nivel más global (cumplimiento de los derechos humanos, la gobernabilidad democrática, la igualdad y la solidaridad entre las personas y los pueblos), en el barrio también trabaja de manera directa a través de un proyecto socioeducativo.

La identificación de los centros socioeducativos ubicados en el barrio se constató con el listado con todos los centros educativos no universitarios y las instituciones de carácter socioeducativo que se encuentran en el Registro de Entidades Ciudadanas del Ayuntamiento de Madrid'.

$1 \quad$ https://sede.madrid.es/portal/site/tramites/menuitem.1f3361415fda829be152e15284f1a5a0/?vgnextoid=a84aef 82e1bed010VgnVCM1000000b205a0aRCRD\&vgnextchannel=c9b8a38813180210VgnVCM100000c90da8c0RCRD 
La elección de esta metodología de análisis se basa en que posibilita caracterizar las estructuras que subyacen a los intercambios materiales o simbólicos que interconectan a un grupo de actores sociales (Diani, 2003). La recogida de información se diseñó ad hoc un cuestionario sociométrico de relaciones instituciones. En este instrumento se solicitó información a cada uno de los representantes de las instituciones educativas sobre diferentes características de las relaciones y sobre el tipo de apoyo que obtenían de ellas (véase Tabla 1).

Tabla 1 . Características de las relaciones. Elaboración propia

\begin{tabular}{|c|c|c|}
\hline \multicolumn{2}{|c|}{ Características de las relaciones } & Descripción \\
\hline \multirow[b]{2}{*}{ Tipo de relación } & Formal & $\begin{array}{l}\text { Relación algún tipo de contrato físico por } \\
\text { escrito. }\end{array}$ \\
\hline & Informal & $\begin{array}{c}\text { Relación no formalizadas legalmente y que } \\
\text { surge de manera espontánea entre las organi- } \\
\text { zaciones }\end{array}$ \\
\hline Antigüedad & $N^{o}$ Años & Tiempo desde el inicio de la relación en años \\
\hline \multirow{3}{*}{ Frecuencia } & Puntual & Menos de una vez a la semana \\
\hline & Frecuente & Una o más veces por semana \\
\hline & Diario & De manera diaria \\
\hline \multirow{4}{*}{ Tipo de apoyo } & Material & Recursos materiales y/o infraestructuras \\
\hline & Económico & Ayudas de carácter monetario \\
\hline & Formativo & Acciones formativas o de asesoramiento \\
\hline & Coordinación & Actuaciones en base a un mismo alumnado \\
\hline
\end{tabular}

Complementariamente, se diseñó el guion de una entrevista semiestructurada que centraba la recogida de información en la percepción general sobre las características del barrio y sus relaciones interinstitucionales y, por otra parte, sobre cuestiones más específicas de las relaciones de propia institución que complementaban el cuestionario anteriormente descrito.

En relaciones a las asociaciones, coincidimos con la definición de esta como organización con nombre propio formada por personas que trabajan de manera organizada e institucionalizada sin tener que recibir una compensación monetaria por ello (Montero, Font y Torcal (eds.), 2006). La información recopilada fue organizada, analizada y representada con Ucinet (Borgatti, 2002) y NETDRAW que se 
encuentran entre los programas específicos de análisis de redes sociales (Borgatti, Everett y Freeman, 2014) y nos permite representar la red gráficamente los datos una vez analizados (Borgatti, 2002). Cada nodo representado corresponde a una de las instituciones del barrio los vínculos entre ellas se representa como con líneas. Este análisis junto con las entrevistas permite conocer qué tipo de relaciones y apoyos se intercambian entre las instituciones. Y comprender cómo son este tipo de apoyos y relaciones y cómo lo viven sus actores, acercándonos aún más a la realidad y necesidades más emergentes del barrio.

El análisis estructural de dichas redes abarca su densidad, sus componentes principales y la distribución estructural de sus actores.

\section{Resultados}

Los datos obtenidos muestran la existencia de una red de relaciones entre las instituciones analizadas. Aunque, posteriormente, este hecho se observa mayor claridad a través la representación gráfica de estas relaciones de apoyo y cooperación, en una primera aproximación hemos querido describir algunas de las características estructurales que presentan las relaciones entre instituciones socioeducativas ubicadas en un contexto desafiante (véase Tabla 2).

Tabla . Características de las relaciones. Elaboración propia

\begin{tabular}{|c|c|c|c|}
\hline \multicolumn{2}{|c|}{ Características Relaciones } & \multirow{2}{*}{$\begin{array}{l}\text { CEIP (\%) } \\
71\end{array}$} & \multirow{2}{*}{$\begin{array}{l}\text { IES (\%) } \\
0\end{array}$} \\
\hline Tipo relación & Formal & & \\
\hline & Informal & 29 & 100 \\
\hline \multirow[t]{3}{*}{ Antigüedad } & + 10 años & 67 & 83 \\
\hline & años & 0 & 0 \\
\hline & -5 años & 33 & 14 \\
\hline \multirow[t]{3}{*}{ Frecuencia } & Puntualmente & 50 & 14 \\
\hline & Frecuentemente & 50 & 83 \\
\hline & Diariamente & 0 & 0 \\
\hline \multirow[t]{2}{*}{ Reciprocidad } & Sí & 86 & 100 \\
\hline & No & 14 & 0 \\
\hline
\end{tabular}




\begin{tabular}{|l|l|l|l|}
\hline \multirow{5}{*}{ Tipo apoyo } & Material & 20 & 12 \\
\cline { 2 - 4 } & Económico & 0 & 0 \\
\cline { 2 - 4 } & Formativo & 20 & 25 \\
\cline { 2 - 4 } & Coordinación & 60 & 63 \\
\hline \multirow{2}{*}{ Participantes } & Equipo directivo/coordinador & 52 & 54 \\
\cline { 2 - 4 } & Docentes/técnico & 32 & 46 \\
\cline { 2 - 4 } & Alumnado/socios & 16 & 0 \\
\hline
\end{tabular}

Los resultados obtenidos en cuanto a la formalización del apoyo indican que éste no parece ser un aspecto determinante a la hora de establecer relaciones de colaboración para el IES que no ha regularizado ninguna de estas relaciones. En contraposición, la entidad asociativa analizada sí se vincula al resto de instituciones a través de convenios formales de colaboración. La mayor parte de las relaciones no son nuevas, el 67\% de las relaciones del CEIP, el 83\% de las relaciones del IES y el $83 \%$ de las relaciones que mantiene la Asociación con el resto de las instituciones socioeducativas del barrio tienen una antigüedad superior a los 10 años.

Aunque los contactos no son diarios en ninguno de los casos, la frecuencia relacional es alta y los contactos son frecuentes en el 50\% de las relaciones del CEIP, en el 83\% de las relaciones del IES y en el 67\% de las relaciones de la Asociación. La reciprocidad también se observa como rasgo característico de gran parte de estas relaciones, así puede afirmarse que todas las relaciones que mantienen tanto el IES como la Asociación y el 83\% de las relaciones que mantiene el CEIP con el resto de las instituciones del barrio son recíprocas, es decir, tiene una doble direccionalidad.

Uno de los aspectos más relevantes en este trabajo, se centraba en describir el tipo de apoyo que se prestan entre sí o comparten las tres instituciones analizadas ha constituido un punto central en nuestra investigación. Las relaciones más frecuentes observadas tienen como base la coordinación interinstitucional para el desarrollo de sus funciones (60\% de las relaciones en las tres instituciones). En este sentido, los informantes pudieron completar esta información argumentado que la falta de recursos y rigidez en la ejecución de las actuaciones por parte de la administración educativa motivan que las instituciones del barrio comiencen a establecer redes entre ellas para dar cobertura a sus necesidades:

Servicios Sociales es muy burocrático (...) y esto hace que el tejido asociativo del barrio cobre sentido porque al no poder dar las respuestas suficientes desde este organismo, somos las asociaciones del barrio las que llegamos a unas coberturas que el propio sistema educativo no llega (E2: P2). 
Ahora siempre en abril hay una reunión en el que agrupa a todas las asociaciones para ver todos los campamentos y todos los recursos que van a tener para el verano, en colegios. Y todos, todos nos los contamos (E1: P10).

A este tipo de relaciones, le siguen las relaciones en las que se comparten formación y apoyo material. La menor incidencia de este tipo de relaciones no indica que las necesidades de apoyo formativo y/o material sean menos relevantes en el barrio. Los propios informantes manifestaron que una de las mayores dificultades a las que se enfrentan es la dependencia económica por parte de las administraciones y las limitaciones que esto les genera:

Porque la falta de recursos hace que te busques la vida para conseguir lo que la administración no te da. Está muy bien reclamar, y está muy hacer huelga y pedir, pedir, pedir y exigir lo que nos debe pertenecer, pero cuando no te la dan y tienes necesidades imperiosas, pues dices, me asocio y busco recursos. (E1: P3)

En las relaciones institucionales no siempre participan todos los miembros de las instituciones e igualmente, suelen estar lideradas por determinadas personas que cumplen una función determinada en la institución. En nuestro trabajo ha podido apreciarse que la mayor parte de las relaciones institucionales que mantienen tanto el CEIP como el IES son lideradas por la persona responsable de la dirección del centro. En cambio, en la estructura de la asociación cobran mayor protagonismo a la hora de establecer estas relaciones el personal técnico de la institución, por encima de la coordinación. Se destaca también la importancia que los informantes otorgan residir en el barrio como una forma de ampliar el compromiso social de estos profesionales: "Los profesores más implicados y que se mueven más por el centro son los que viven en el barrio" (E1:P8).

Del mismo modo, que la estabilidad en el puesto de trabajo hace que las relaciones sean más fluidas y se generen lazos de confianza entre las personas que vinculan las instituciones:

Llevamos muchos años juntos y te atienden por WhatsApp, por email, o vas. La coordinación es muy alta. Una de las cosas que es muy positiva es que la continuidad de los agentes que hay es muy alta. Entonces los conoces desde hace más de 10 años. Eso es muy positivo. Y con los centros, depende de la relación que tengas, porque al final depende de la persona y depende de la buena voluntad. No es un tema protocolario, es un tema de intencionalidad(E2:P2).

Como se ha avanzado con anterioridad, la representación gráfica de las relaciones evidencia la existencia de una red de apoyo informal que incluye relaciones tanto a centros educativos de Educación 
Infantil, Primaria y Secundaria como a asociaciones no gubernamentales sin ánimo de lucro que realizan una función socioeducativa en el barrio. En consecuencia, cada una de las redes tiende a conectar entre sí a una parte de los actores.

Como puede apreciarse, la densidad de las redes es relativamente baja en los tres casos. Para el CEIP la densidad es del 18,1\%, para el IES del 16,7\% y para la Asociación del 15,3\%. A pesar de ello, es necesario relativizar este valor ya que lo más importante acerca de la densidad de una red va más allá de su valor en sí y es necesario relativizar su valor de acuerdo con el tipo de estructura propio de cada red (Garavito, 2012).

Ilustración 1. Relaciones del CEIP

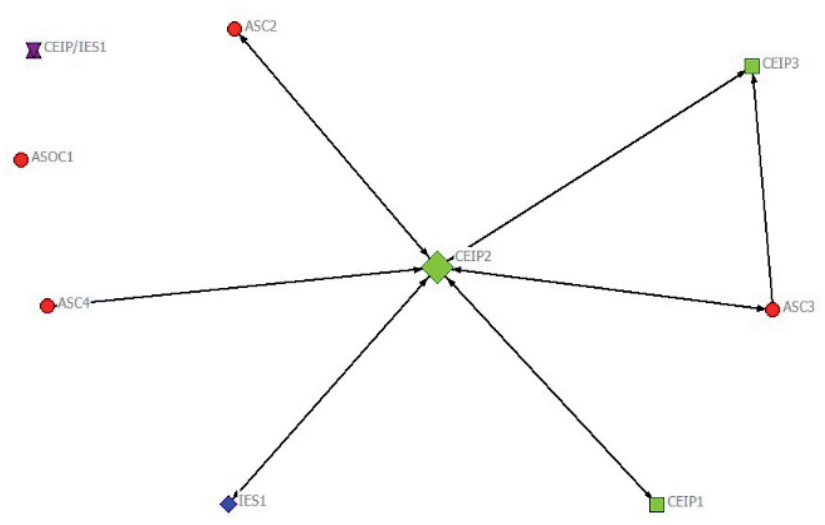

Ilustración 2. Relaciones del IES

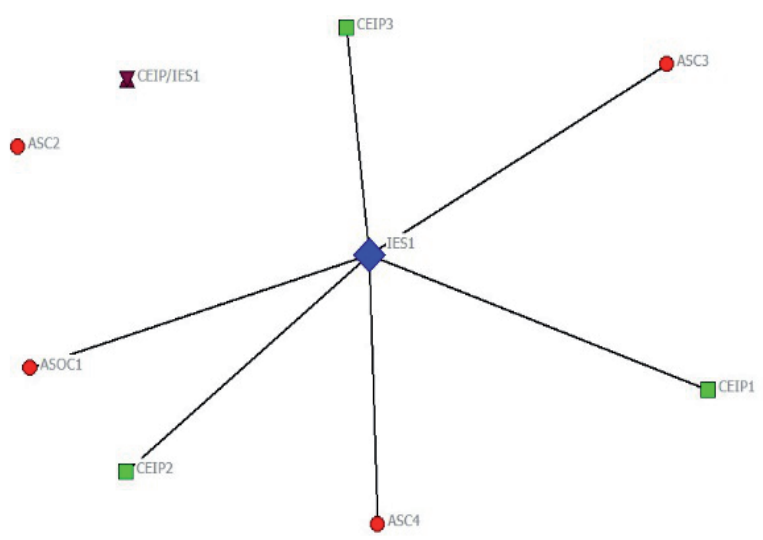


Ilustración 3. Relaciones de la ONG

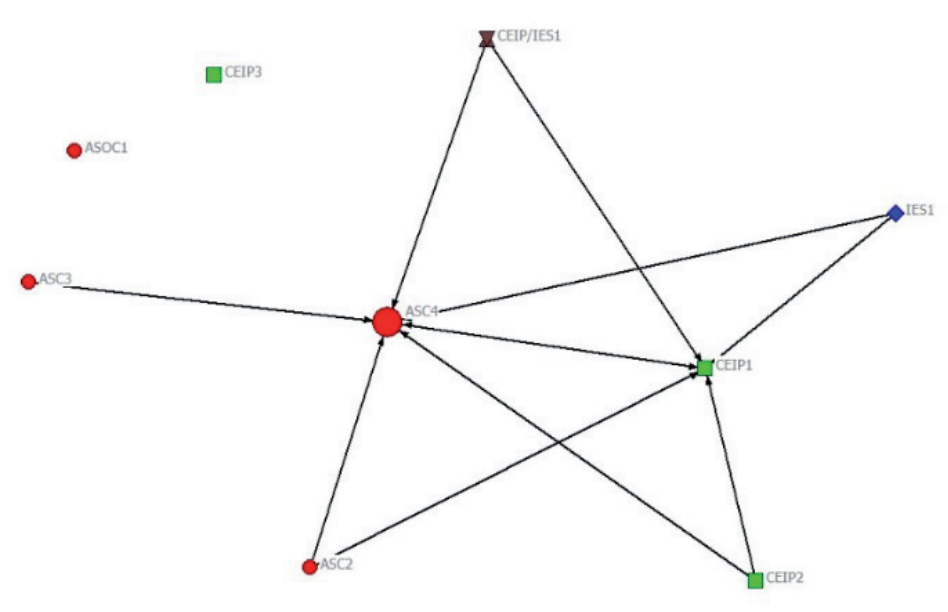

\section{Discusión y conclusiones}

Es importante resaltar que los resultados obtenidos hasta el momento son una primera aproximación de una investigación piloto.

A partir de estos primeros resultados, consideramos que la estructura de red social entre las instituciones socioeducativas del barrio surge como respuesta urgente a las demandas sociales y educacionales del alumnado y sus familias, y de la falta de recursos que no son aportados por las administraciones públicas locales y estatales.

Los insuficientes recursos que la administración pública ofrece al barrio promueven una necesidad de cooperación de las instituciones que se encuentran en él. Así es como se crea una red de información y apoyo de instituciones sociales y educativas que se apoyan unas a otras para reducir las demandas y necesidades del alumnado.

Las instituciones socioeducativas reclaman una mayor atención por parte de las diferentes administraciones públicas. No solamente demandan que se tenga presente que el barrio tiene características propias por formar parte de un contexto desafiante, sino que también se requiere reconocer que esta situación afecta a las personas que viven en el mismo. Como consecuencia de la falta de atención por parte de dichas administraciones, para poder cubrir todo tipo de necesidades que se manifiestan, son las diferentes entidades que trabajan día a día en el barrio e intentan buscar los recursos que necesitan para garantizar una cobertura de calidad a las necesidades de las personas que viven en el mismo.

Con estos resultados nos reafirmamos también en la definición de capital social que hacen autores como Bordieu (1980) o Lin (1999), una definición del concepto relacionada con los recursos que se vuel- 
ven accesible gracias a la conexión de estas redes y no de las propias redes por sí. Por otro lado, otro estudio demostró la importancia de la conexión entre instituciones relacionadas con la salud (Faust, Christens, Sparks Hilgendorf, 2015) afirmando que esta conexión mejoraba en la intervención. Es similar a lo que vemos en esta investigación relacionada con entidades socioeducativas: su conexión permite la mejora de calidad de vida de las personas que viven el barrio a pesar del vacío institucional que sufren.

\section{Referencias}

Alguacil J., Camacho, J., y Hernández, H. (2014). La vulnerabilidad urbana en España. Identificación y evolución de los barrios vulnerables. Empiria. Revista de metodología de ciencias sociales, (27), 73-94.

Alguacil, J. (2011, junio). Sobre la vulnerabilidad urbana. Presentación en Jornada La vulnerabilidad urbana en España: Instrumentos para el análisis y políticas para la acción I Foro de Debates: Ciudad y Territorio. Simposio realizado en la Secretaría de Estado de Vivienda y Actuaciones Urbanas del Ministerio de Fomento, Madrid, 30 de junio de 2011.

Beckett, L. y Wrigley, T. (2014). Overcoming stereotypes, discovering hidden capitals. Improving Schools, 17(3), 217-230.

Blanco, G. (2006). La equidad y la inclusión social: uno de los desafíos de la educación y la escuela hoy. REICE. Revista Iberoamericana sobre Calidad, Eficacia y Cambio en Educación, 4(3), 1-15.

Borgatti, S. P. (2002). NetDraw software for network visualization. Lexington, KY: Analytic Technologies, 95 .

Borgatti, S. P., Everett, M. G., y Freeman, L. C. (2014). Ucinet. In Encyclopedia of social network analysis and mining (pp. 2261-2267). Springer New York.

Bourdieu, P. (1980). L'identité et la représentation [Éléments pour une réflexion critique sur l'idée de région]. Actes de la recherche en sciences sociales, 35(1), 63-72.

Chapman, C. y Harris, A. (2004). Improving schools in difficult and challenging contexts: Strategies for improvement. Educational Research, 46(3), 219-228.

Civís, M; Longás, J. (2015). La colaboración interinstitucional como respuesta al desafío de la inclusión socioeducativa. Análisis de 4 experiencias de trabajo en red a nivel local en Cataluña. Educación XX1, Enero-Junio, 213-236. 
Comisión Europea (2017). Documento de reflexión sobre la dimensión social de Europa. Comisión Europea: Bruselas. Disponible: https://ec.europa.eu/commission/sites/beta-political/files/reflection-paper-social-dimension-europe_es.pdf

Corbett, D., Wilson, B., y Williams, B. (2002). Effort and excellence in urban classrooms. New York: Teachers College Press.

Corraliza, J. A. (2014). Vida urbana y experiencia social: variedad, cohesión y medio ambiente. Boletín $C F+S$, (15).

Diani, M. (2003). Leaders or brokers? Positions and influence in social movement networks. Social movements and networks: Relational approaches to collective action. Oxford: Oxford University Press

Dyson, L. (2004). The effect of neighbourhood poverty and low-income on the school context: Teachers' expectations for achievement and rating of achievement motivation of elementary school children. Canadian society for the study of education. Winnipeg: Canada.

Faust, V., Christens, B. D., Sparks, S. M. A., y Hilgendorf, A. E. (2015). Are networks of organizational collaboration associated with organizational learning and effectiveness? Psychosocial Intervention, 24 (3), 125-131.

Fernández, M. T., Tuset, A. M., Pérez, R. E., y García, C. (2013). Prácticas educativas y creencias de profesores de secundaria pertenecientes a escuelas de diferentes contextos socioeconómicos. Perfiles educativos, 35(139), 40-59.

Garavito, L. (2012). Las redes informales inter-oganizacionales en la movilización por el agua en la Cuenca de México, 2005-2009. Revista hispana para el análisis de redes sociales, 23 (8), 233-260.

Gray, J. (2000) Causing concern but improving: a review of schools' experience: London: DfEE.

Gremmen, M. C., Dijkstra, J. K., Steglich, C., y Veenstra, R. (2017). First selection, then influence: Developmental differences in friendship dynamics regarding academic achievement. Developmental psychology, 53(7), 1356-1370.

Gualda, E. y Márquez, C. (2010). The role of social networks in the small Cameroons community in Huelva (Spain). In the way to inclusion. En E. Lucio-Villegas (Ed.) Transformingresearching communities (pp. 127-140). Barcelona: El Masnou. 
Harris, A. (2002). Effective leadership in schools facing challenging contexts. School Leadership \& Management, 22(1), 15-26.

Harris, A. y Chapman, C. (2004). Improving schools in difficult contexts: Towards a differentiated approach. British Journal of Educational Studies, 52(4), 417-431.

Hayes, D., Jonhston, K. y King, A. (2009). Creating enabling classroom practices in high poverty contexts: the disruptive possibilities of looking in classrooms. Pedagogy, Culture \& Society, 17(3), 251-264.

Horgan, G. (2009). ‘That child is smart because he's rich': the impact of poverty on young children's experiences of school. International Journal of Inclusive Education, 13(4), 359-376.

Kauppinen, T. M. (2008). Schools as mediators of neighbourhood effects on choice between vocational and academic tracks of secondary education in Helsinki. European Sociological Review, 24(3), 379-391.

Lin, N. (1999). Building a network theory of social capital. Connections, 22 (1), 28-51

Lomnitz, L. (1977). Networks and marginality. Nueva York: Academic Press.

Longàs, J., Civís, M. y Riera, J. (2008). Asesoramiento y desarrollo de redes socioeducativas locales. Funciones y metodología. Cultura y Educación, 20(3), 303-324.

López de Lucio, R. (1988). Génesis y remodelación de una parcelación periférica madrileña: el Pozo del Tío Raimundo (Vallecas). Ciudad y Territorio, (76), 55-70.

López, N., D’Alessandre, V. y Pla, J. (2006). Educación y desigualdad social. Buenos Aires: Ministerio de Educación, Ciencia y Tecnología de la Nación

Luego-Marín, D. R. (2011). Análisis de redes sociales en el mundo rural: guía inicial. Revista de estudios sociales, (38), 129-142.

Luke, D. A. (2005). Getting the big picture in community science: Methods that capture context. American Journal of Community Psychology, 35, 185-200. http://dx.doi.org/10.1007/ s10464-005-3397-z PMid:15909794-131.

Matilla-García, M. (2017). Aspectos cuantitativos del análisis de redes sociales. Papeles de economía española, 152, 2-13. 
Montero J.R., Fontj., Torcal, M. (eds.). 2006. Ciudadanos, asociaciones y participación en España. Madrid: Centro de Investigaciones Sociológicas.

Murillo, F. J., y Martínez-Garrido, C. (2018). Magnitud de la Segregación escolar por nivel socioeconómico en España y sus Comunidades Autónomas y comparación con los países de la Unión Europea. RASE: Revista de la Asociación de Sociología de la Educación, $11(1), 37-58$.

Murillo, F., Belavi, G., y Pinilla Rodríguez, L. (2018). Segregación escolar público-privada en España. Papers. Revista De Sociología, 103(3), 307-337. doi:http://dx.doi.org/10.5565/ rev/papers. 2392

Nancy, J.L. (1991). The Inoperative Community. Minneapolis: University of Minnesota Press.

Nancy, J.L. (2000). Being Singular Plural. Stanford, CA: StanfordUP.

O'Connor, M., Hales, E., Davies, J. y Tomlinson, S. (1999). Hackney Downs: The school that dared to fight. Hackney Downs, London: Cassell.

Paredes, A., Vitaliti, JM, Aguirre, J. Strafile, S. y Jara, C. Tipos de apoyo y la digitalización de las redes personales. El uso de Facebook de adolescentes rururbanos de Mendoza (Argentina). Redes. Revista Hispana para el Análisis de Redes Sociales, 26, (1) 97-123

Pereda, C. (2003). Escuela y comunidad. Observaciones desde la teoría de sistemas sociales complejos. REICE: Revista Electrónica Iberoamericana sobre Calidad, Eficacia y Cambio en Educación, 1 (1), 1-15.

Potter, D., Reynolds, D. y Chapman, C. (2002) School improvement for schools facing challenging circumstances: a review of research and practice, School Leadership and Management, 12(3), 243-256.

Requena, F. (1989). El concepto de red social. REIS. Revista Española de Investigaciones Sociológicas, 48(89), 137-152

Santos, M. (2010). Análisis de redes sociales y rendimiento académico: lecciones a partir del caso de los Estados Unidos. Debates en sociología, (35), 7-44

Sanz, L. (2003). Análisis de redes sociales o cómo representar las estructuras sociales subyacentes. Apuntes de ciencia y tecnología, 7, pp. 21-29 
Shin, H., y Ryan, A. M. (2014). Early adolescent friendships and academic adjustment: Examining selection and influence processes with longitudinal social network analysis. Developmental Psychology, 50(11), 2462-2472.

Sipi, R. (2000). Las asociaciones de mujeres, ¿agentes de integración social? Papers: Revista de sociología, 60, 355-364.

Smith, J. (2014). Improving schools in poor areas: It's not about the organisation, structures and privatisation, stupid! Improving Schools, 17(3), 231-240.

Stiglitz, J. E. (2012). El precio de la desigualdad: el 1\% de población tiene lo que el 99\% necesita. Madrid: Taurus.

Stoll, L. y Myers, K. (1998) Introduction, in: L. Stoll \& K. Myers (Eds). No quick fixes: perspectives on schools in difficulty (London, Falmer Press).

Thrupp, M. (2011) Review of 'Schooling in disadvantaged communities. Playing the game from the back of the field', Journal of Education Policy, 26(5), 732-733.

Thrupp, M. y Lupton, R. (2006). Taking school contexts more seriously: the social justice challenge. British Journal of Educational Studies, 54(3), 308-328.

Vermeij, L., Van Duijn, M. A., y Baerveldt, C. (2009). Ethnic segregation in context: Social discrimination among native Dutch pupils and their ethnic minority classmates. Social Networks, 31(4), 230-239.

Zazo, A., Ardines, S. y Castro, E. (2015). Redes de colaboración de las unidades de investigación de la Universidad de Panamá: investigación, desarrollo e innovación. Revista hispana para el análisis de redes sociales, 26(2), 84-117. 